\section{Diabetes and Overweight/Obesity Are Independent, Nonadditive Risk Factors for In-Hospital Severity of COVID-19: An International, Multicenter Retrospective Meta-analysis}

https://doi.org/10.2337/dc20-2676

\section{OBJECTIVE}

Obesity is an established risk factor for severe coronavirus disease 2019 (COVID-19), but the contribution of overweight and/or diabetes remains unclear. In a multicenter, international study, we investigated if overweight, obesity, and diabetes were independently associated with COVID-19 severity and whether the BMIassociated risk was increased among those with diabetes.

\section{RESEARCH DESIGN AND METHODS}

We retrospectively extracted data from health care records and regional databases of hospitalized adult patients with COVID-19 from 18 sites in 11 countries. We used standardized definitions and analyses to generate site-specific estimates, modeling the odds of each outcome (supplemental oxygen/noninvasive ventilatory support, invasive mechanical ventilatory support, and in-hospital mortality) by BMI category (reference, overweight, obese), adjusting for age, sex, and prespecified comorbidities. Subgroup analysis was performed on patients with preexisting diabetes. Sitespecific estimates were combined in a meta-analysis.

\section{RESULTS}

Among 7,244 patients (65.6\% overweight/obese), those with overweight were more likely to require oxygen/noninvasive ventilatory support (random effects adjusted odds ratio [aOR], 1.44; 95\% Cl 1.15-1.80) and invasive mechanical ventilatory support (aOR, 1.22; 95\% Cl 1.03-1.46). There was no association between overweight and in-hospital mortality (aOR, 0.88; 95\% Cl 0.74-1.04). Similar effects were observed in patients with obesity or diabetes. In the subgroup analysis, the aOR for any outcome was not additionally increased in those with diabetes and overweight or obesity.

\section{CONCLUSIONS}

In adults hospitalized with COVID-19, overweight, obesity, and diabetes were associated with increased odds of requiring respiratory support but were not associated with death. In patients with diabetes, the odds of severe COVID-19 were not increased above the $\mathrm{BMI}$-associated risk.
Danielle K. Longmore, ${ }^{1,2,3}$

Jessica E. Miller, ${ }^{1,4}$ Siroon Bekkering, ${ }^{1,5}$

Christoph Saner, ${ }^{1,6}$ Edin Mifsud, ${ }^{1,7}$

Yanshan Zhu, ${ }^{8}$ Richard Saffery, ${ }^{1,4}$

Alistair Nichol, ${ }^{9,10,11}$ Graham Colditz, ${ }^{12}$

Kirsty R. Short, ${ }^{8}$ and David P. Burgner, ${ }^{1,3,4,13}$

on behalf of the International BMI-COVID consortium*

${ }^{1}$ Murdoch Children's Research Institute, The Royal Children's Hospital, Parkville, Victoria, Australia

${ }^{2}$ Menzies School of Health Research, Charles Darwin University, Darwin, Australia

${ }^{3}$ Infectious Diseases Unit, Department of General Medicine, The Royal Children's Hospital, Parkville, Victoria, Australia

${ }^{4}$ Department of Paediatrics, Melbourne University, Parkville, Victoria, Australia

${ }^{5}$ Department of Internal Medicine, Radboud Institute for Molecular Life Sciences, Radboud University Medical Center, Nijmegen, the Netherlands ${ }^{6}$ Pediatric Endocrinology, Diabetology and Metabolism, Department of Pediatrics, University Hospital Inselspital, University of Bern, Bern, Switzerland

${ }^{7}$ World Health Organization Collaborating Centre for Reference and Research on Influenza, Doherty Institute, Melbourne, Australia

${ }^{8}$ School of Chemistry and Molecular Biosciences, The University of Queensland, Brisbane, Australia ${ }^{9}$ Department of Intensive Care, Alfred Health, Melbourne, Australia

${ }^{10}$ Australian and New Zealand Intensive Care Research Centre, Monash University, Melbourne, Australia

${ }^{11}$ University College Dublin Clinical Research Centre, St Vincent's Hospital, Dublin, Ireland

${ }^{12}$ Washington University, St. Louis, MO

${ }^{13}$ Department of Paediatrics, Monash University, Clayton, Victoria, Australia

Corresponding authors: David P. Burgner, david. burgner@mcri.edu.au, and Danielle K. Longmore, danielle.longmore@mcri.edu.au

Received 29 October 2020 and accepted 14 January 2021

This article contains supplementary material online at https://doi.org/10.2337/figshare.13616024.

D.K.L. and J.E.M. contributed equally as first authors, and K.R.S. and D.P.B. contributed equally as senior authors.

*A complete list of International BMI-COVID consortium members is included in the supplementary material online.

This article is part of a special article collection available at https://care.diabetesjournals.org/ collection/diabetes-and-COVID19.

(C) 2021 by the American Diabetes Association. Readers may use this article as long as the work is properly cited, the use is educational and not for profit, and the work is not altered. More information is available at https://www.diabetesjournals .org/content/license. 
In the first 6 months of the coronavirus disease 2019 (COVID-19) pandemic (until June 30,2020$),>10$ million people had been infected with severe acute respiratory syndrome coronavirus 2 (SARSCoV-2) and $>500,000$ COVID-19-related deaths had been recorded (1), but striking variation in clinical severity and outcomes remains. Identifying risk factors associated with more severe COVID-19 is essential for optimizing individual treatment, resource allocation, and prioritizing immunization distribution. Obesity has emerged as an important risk factor for severe COVID-19 (2), but several key questions remain unanswered (3).

First, most studies to date have focused on individuals with obesity (BMI $\geq 30 \mathrm{~kg} / \mathrm{m}^{2}$ ) (4), but the specific contribution of overweight (BMI between $\geq 25$ and $<30$ ) to severe COVID-19 has only been investigated in a few studies, which have reported inconsistent results (510). This is a significant knowledge gap, because $40 \%$ of the global population is overweight, in addition to the $13 \%$ living with obesity (11). Second, most studies are single-center analyses and are unlikely to be globally representative, given the marked intercountry variation in overweight and obesity (12). This shortcoming has partially been addressed by meta-analyses, but these rarely include individuals who are overweight (2). Finally, both overweight and obesity frequently occur with other comorbidities, particularly type 2 diabetes (13). However, many studies have either not adjusted for these covariables or the regression models used did not allow clinical translation of findings (3). Specifically, a key clinical question is whether patients with both diabetes and higher BMI have a higher risk of severe COVID19 compared with those with diabetes and $\mathrm{BMI}$ in the normal range.

In the present study, we aimed to address these unresolved questions by performing an international, retrospective, multisite analysis of 7,244 hospitalized patients with COVID-19 from 18 sites in 11 countries. We used common definitions and analyses to delineate whether overweight, obesity, and diabetes are independent risk factors for respiratory support and in-hospital mortality. In patients with diabetes, we also investigated the association between BMI category and COVID-19 severity.

\section{RESEARCH DESIGN AND METHODS}

\section{Study Design}

We conducted an international, multicenter, retrospective analysis of hospitalized patients with COVID-19 from a total of 69 hospitals in 11 countries (Supplementary Table 1) from 17 January 2020 to 2 June 2020. Data from 69 hospitals were collated to form 18 sites that each provided site-specific outcomes and estimates. We modeled the odds of in-hospital respiratory support (ie, supplemental oxygen/noninvasive ventilatory support, invasive mechanical ventilatory support) and in-hospital mortality by BMI category, adjusting for age, sex, and prespecified comorbidities, as described later in this section. A protocol was finalized on 20 April 2020 (see Supplementary Material) prior to commencement of the study. The study was conducted in accordance with Good Clinical Practice guidelines, local regulations, and the ethical principles described in the Declaration of Helsinki. Ethical approval was obtained at the coordinating center (Murdoch Children's Research Institute [MCRI], Royal Children's Hospital, Melbourne, Australia; approval no. HREC 63887), and local approvals were obtained at participating sites, depending on local regulations. Informed consent was not required.

\section{Data Source}

We analyzed deidentified data from existing collections of hospital data and regional databases, including the Norwegian Intensive Care and Pandemic Registry, Norway; and Washington University, St. Louis, Missouri (see Supplementary Appendix for participating sites and investigators). Data from smaller contributing hospitals were collected for clinical auditing processes approved by local hospitals and in accordance with local regulations. Each site followed a standardized protocol for data coding and analysis to generate site-specific estimates for each study population (Supplementary Material). Deidentified data from hospitals in Austria, Singapore, the Netherlands, Switzerland, and Indonesia were exported to the coordinating center (MCRI) for generation of sitespecific estimates. All transfer of data and site-specific estimates to the MCRI was subject to a data transfer agreement. Statisticians at the MCRI completed the meta-analyses.

\section{Data Collection}

Study participants were aged $\geq 18$ years, admitted to hospital with COVID-19 (confirmed by PCR for SARS-CoV-2), had height and weight recorded on admission to participating sites with local approval to participate. The period for data collection from individual sites varied (Supplementary Table 1).

Information regarding participant demographic variables (i.e., age, sex), BMI, pre-existing medical conditions, clinical variables including intensive care unit admission, and treatment including oxygen and noninvasive ventilatory support and mechanical ventilatory support were identified. Supplemental oxygen was defined as the provision of oxygen via nasal canulae or face mask. Noninvasive ventilatory support was defined as the use of a device providing continuous positive airway pressure or bilevel positive airway pressure. Cardiovascular disease was defined as preexisting, physician-diagnosed coronary heart disease, ischemic stroke, heart failure, and/or peripheral vascular disease. Diabetes was defined as preexisting diabetes (including type 1 or 2 ). In all countries, type 2 diabetes was diagnosed according to the American Diabetes Association guidelines or local guidelines with the same diagnostic criteria as the American Diabetes Association guidelines. For three sites only (Cape Town, South Africa; Lausanne, Switzerland; and Ticino, Switzerland), a small number of patients were included who were first diagnosed with diabetes during their admission with COVID-19. Preexisting respiratory conditions and hypertension were defined as physician-diagnosed and currently on treatment. Data cleaning was performed for out-of-range values, inconsistent data, and repeated participant entries. Central source data verification was not feasible for this study, because coding was performed by the individual participating centers.

\section{Statistical Analysis}

All analyses were conducted as outlined in our protocol (Supplementary Material). Participant data are presented as frequency, reported as percentage. Each site (or the MCRI) followed a standardized protocol for data coding and analysis to generate site-specific estimates from each study population, modeling the odds of each outcome by BMI (calculated 
as weight $[\mathrm{kg}]$ divided by height squared $\left.\left[\mathrm{m}^{2}\right]\right)$ category (Supplementary Material). BMI was categorized as underweight ( $\geq 12$ to $<18$ ), normal ( $\geq 18$ to $<25$ [the referent]), overweight ( $\geq 25$ to $<30$ ), and obese $(\geq 30)$. In sensitivity analyses for Asian populations, respective BMI categories were based on the following ranges: $\geq 12$ to $<18.5, \geq 18.5$ to $<24$ (referent), $\geq 24$ to $<28$, and $\geq 28$ (14). Logistic regression was used to model the association between BMI category and the use of in-hospital respiratory therapies (listed previously in this section) and in-hospital mortality.

All models estimated crude (unadjusted) and adjusted odds ratios. Two levels of adjustments were made. The first level, available for all sites, included age, sex, preexisting cardiovascular disease, diabetes, preexisting respiratory conditions, and hypertension. The second level of adjustments included the first level of adjustments plus current smoking status and/or race/ethnicity, depending on data availability. The second level was available for only five sites. The crude and adjusted (first-level) models were run on data from a subgroup of patients with preexisting diabetes. No adjustment was made for multiple comparisons. Covariables had few missing data and no imputations were warranted. Site-specific adjusted estimates for BMI category, each independent covariable included in the adjusted models, and the diabetes subgroup estimates were combined in meta-analyses.

Summarized estimates included fixed and random effects models (15). Random effects estimates are presented in the text. Of note, the Los Angeles, New York, and Cape Town sites were not included in analysis of supplemental oxygen/ noninvasive ventilatory support, because nearly all hospitalized patients received supplemental oxygen per local policies. Data on supplemental oxygen were not available for Austria, Norway, or Amphia (the Netherlands). Variations to the preplanned analysis were made because there were insufficient data available from the majority of sites. The outcomes not analyzed included intensive care unit length of stay, hospital length of stay, and extracorporeal membrane oxygenation use (Supplementary Material). Sitespecific analyses were performed in SAS (SAS Institute, Cary, NC), Stata (StataCorp, College Station, TX) or R studio (PBC,
Boston, MA) (16). Meta-analyses were performed in Stata SE, version 16.0 (17).

\section{RESULTS}

Characteristics of Patients Included in the Study

A total of 7,244 patients from 18 sites ( $n=69$ hospitals) in 11 countries were included in this study of hospitalized patients with COVID-19 (Supplementary Tables 1 and 3). Among these, 60.1\% were male and $51.7 \%$ were older than 65 years. Overall, $34.8 \%$ were overweight and $30.8 \%$ obese; however, there was considerable variability across different individual countries and sites. Prevalence of obesity for each site country is provided in Supplementary Table 2. The rates of comorbidities and the frequency of outcomes varied across sites (Supplementary Tables 3 and 4). Prevalence of diabetes varied from $7 \%$ in Guangdong Province, China, to $46 \%$ in St. Louis, Missouri. Prevalence of diabetes among those of normal weight ranged from $6 \%$ in Milan Sacco, Italy, to $39 \%$ in Cape Town, South Africa. Prevalence of diabetes among those who were overweight or obese ranged from $7 \%$ to $5 \%$, respectively, in Guangdong Province, China, to $47 \%$ and $53 \%$, respectively, in St. Louis, Missouri.

Association of Overweight, Obesity, and Supplemental Oxygen/

Noninvasive Ventilatory Support, Mechanical Ventilatory Support, and In-Hospital Death

Compared with normal weight, overweight and obesity were associated with increased odds of supplemental oxygen/ noninvasive ventilatory support (random effects adjusted odds ratio (aOR), 1.44 [95\% Cl 1.15-1.80], $P=0.02$; and aOR, 1.75 [95\% Cl 1.33-2.30], $P<0.01$ ), respectively (Fig. 1). Obesity was associated with a $73 \%$ increase in odds for invasive mechanical ventilatory support (aOR, 1.73; 95\% Cl 1.29-2.32; $P<0.01$ ) (Fig. 2), and a more modest association was observed for overweight (aOR, 1.22; 95\% Cl 1.03-1.46; $P=0.02$ ). Data on this outcome were not available from Amphia (the Netherlands).

Overweight was not associated with an increase in odds for in-hospital mortality (aOR, 0.88; 95\% Cl 0.74-1.04; $P=$ 0.13 ) (Fig. 3). Obesity was also not associated with an increase in odds of in-hospital mortality, with confidence limits including the null (aOR, 1.23; 95\%
$\mathrm{Cl} 0.92-1.64 ; P=0.17)$. The low number of participants in the underweight group $(n=$ 162) precluded calculation of robust odds ratios. The $I^{2}$ statistic, which describes the percentage of variation across studies that is due to heterogeneity rather than chance, was $43.6 \%$ and $53.7 \%$ among the obese groups for invasive mechanical ventilatory support and in-hospital mortality, respectively, suggesting modest heterogeneity across studies. Unadjusted sitespecific odds ratios are presented in Figs. 1-3.

For the Chinese, Indonesian, and Singaporean sites, odds ratios varied slightly depending on whether the standard or Asian country-specific BMI categories were used. The variation did not meaningfully affect the summarized metaanalysis estimates (Supplementary Table 4). Additional adjustments for current smoking and race/ethnicity, where data were available, had little impact on the odds ratios (Supplementary Table 5).

\section{Association of Diabetes With} Supplemental Oxygen/Noninvasive Ventilatory Support, Mechanical Ventilatory Support, and In-Hospital Death

Compared with patients without diabetes, those with diabetes had an increased odds of needing mechanical ventilatory support in random effects models adjusted for all covariables, including BMI category and comorbidities (aOR 1.21; 95\% Cl 1.03-1.41; $P=0.02$ ) (Supplementary Fig. 1). There was no increase in odds of noninvasive respiratory support or inhospital mortality in those with diabetes (Supplementary Fig. 1). In addition to diabetes, other host factors previously associated with severe COVID-19 (i.e., increased age, male sex, preexisting cardiovascular disease, and chronic respiratory disease) (18) were each independently associated with an increased risk of one or more of the selected study outcomes (Supplementary Figs. 2-4).

\section{Among Patients With Diabetes,} Increased BMI Did Not Increase the Risk of Severe COVID-19 Outcomes To further inform patient care, we next performed a subgroup analysis of individuals with diabetes. Specifically, we investigated if BMI category among those with diabetes was associated with the selected COVID-19 outcomes. Strikingly, there was no association between 


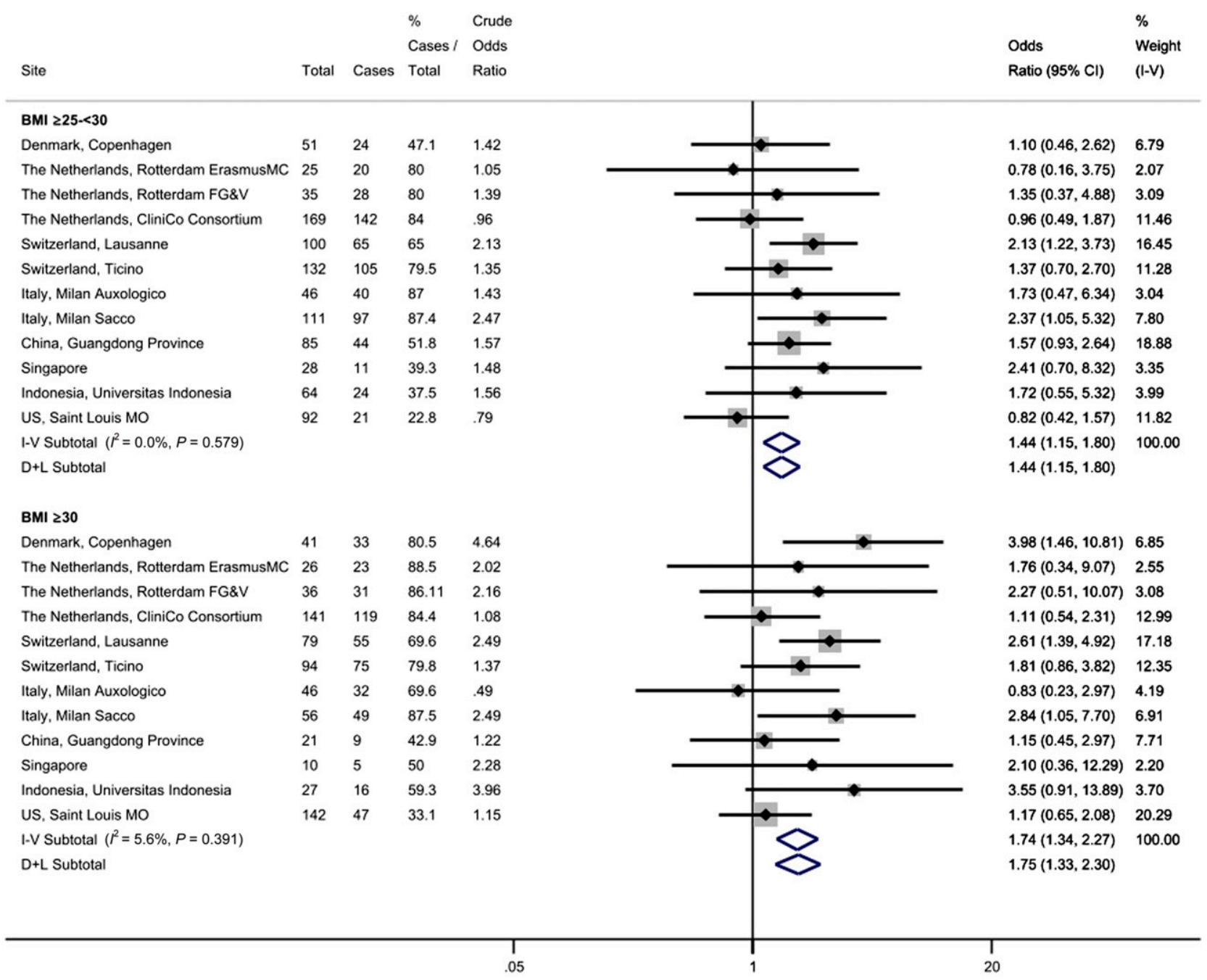

Figure 1-Meta-analysis odds ratios for requirement of supplemental oxygen/noninvasive ventilatory support by BMI category. Models were adjusted for age ( $<65, \geq 65$ years), sex (male/female), preexisting cardiovascular disease (yes/no), diabetes (yes/no), preexisting respiratory conditions (yes/no), and hypertension (yes/no). The reference BMI is in the normal range (i.e., $\geq 18$ to $<25$ ). The $95 \% \mathrm{Cls}$ of the odds ratios were not adjusted for multiple testing and should not be used to infer definitive effects. Data from Norway; Amphia (in the Netherlands); Austria; South Africa; University of California, Los Angeles, California; Cornell University, Ithaca, New York, were not included in this model, because data were either not available for this outcome or all patients received the therapy. D+L, DerSimonian and Laird random effects model; FG\&V, Franciscus Gasthuis \& Vlietland; I-V, inverse-variance weighted fixed effects model; MC, medical center.

overweight or obesity and supplemental oxygen use/noninvasive ventilatorysupport (aOR $1.04[95 \% \mathrm{Cl} 0.54-2.00], P=$ 0.91 ; and 1.29 [95\% $\mathrm{Cl} 0.68-2.46], P=$ 0.44 , respectively), invasive mechanical ventilatory support (aOR $0.67[95 \% \mathrm{Cl}$ $0.40-1.12], P=0.10$; and $1.25[95 \% \mathrm{Cl}$ $0.62-2.53$ ], $P=0.73$, respectively), or in-hospital mortality (aOR $0.79[95 \% \mathrm{Cl}$ $0.52-1.20], P=0.28$; and $1.14[95 \% \mathrm{Cl}$ $0.61-2.13$ ], $P=0.52$, respectively) in those with preexisting diabetes (Fig. 4). In this subgroup analysis, the sample size was reduced and resulted in wide Cls.

\section{CONCLUSIONS}

In this large, international, multicenter study of patients hospitalized with
COVID-19, overweight was associated overall with an increased requirement of respiratory support. The association between overweight and in-hospital mortality was not statistically significant. Similar trends were observed in patients with obesity. In addition to the associations with BMI, diabetes was independently associated with increased COVID19 severity but not death. Importantly, among patients with diabetes, overweight/ obesity were not associated with an increased risk of severe COVID-19.

The data presented here are consistent with those of previous studies that reported not only obesity but also advanced age, male sex, and preexisting cardiovascular, metabolic, and respiratory disease were associated with worse outcomes with COVID-19 (19). In the present study, neither overweight/obesity nor diabetes was associated with in-hospital mortality. Although previous analyses have suggested that obesity increases the mortality risk associated with COVID$19(4,8,20)$, these studies did not necessarily make adjustments for age, sex, and other comorbidities as we did in the present study, or only found an effect on death for those with more severe obesity (BMI > 35) (21). The data presented here are consistent with previous findings that an elevated BMI is associated with an increased requirement for respiratory support $(5,22-24)$ and that diabetes in patients with COVID-19 is not 


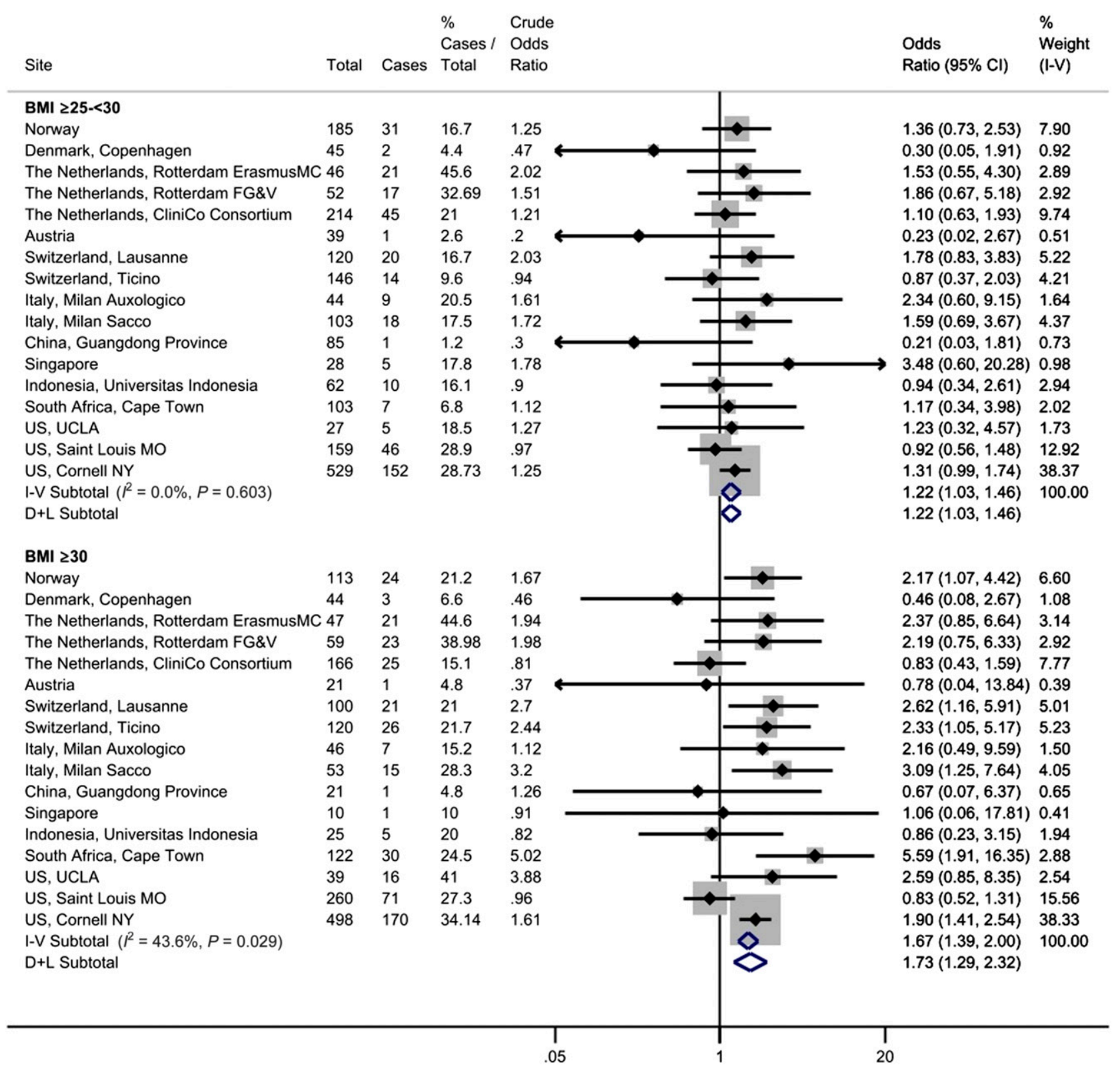

Figure 2-Meta-analysis odds ratios for invasive mechanical ventilatory support by BMI category. Models were adjusted for age ( $<65$, $\geq 65$ years), sex (male/female), preexisting cardiovascular disease (yes/no), diabetes (yes/no), preexisting respiratory conditions (yes/no), and hypertension (yes/no). The reference BMI is in the normal range (i.e., $\geq 18$ to $<25$ ). The $95 \% \mathrm{Cls}$ of the odds ratios have not been adjusted for multiple testing and should not be used to infer definitive effects. Data from Amphia (in the Netherlands) were not available for invasive mechanical ventilatory support. $D+L$, DerSimonian and Laird random effects model; FG\&V, Franciscus Gasthuis \& Vlietland; I-V, inverse-variance weighted fixed effects model; US UCLA, University of California, Los Angeles, California.

significantly associated with in-hospital mortality after appropriate adjustment (25).

The mechanisms underlying the association between BMI and COVID-19 severity likely reflect a dysregulated host response, resulting in heightened inflammation and/or a suboptimal antiviral response. There are a number of relevant immunomodulatory effects of overweight/ obesity, including chronic systemic inflammation (26), reduced production of type I interferons (27), reduced antigen presentation (28), complement activation (29), and/or suboptimal T-cell responses (30). Moreover, these immunomodulatory effects may be compounded by the reduced functional respiratory capacity in individuals with overweight/obesity, which may lead to a lower threshold for noninvasive or invasive respiratory support (31).

It is likely that the independent role identified for diabetes in COVID-19 severity reflects the role of hyperglycemia in severe respiratory disease. Indeed, data from Denmark suggest that each $1 \mathrm{mmol} / \mathrm{L}$ increase in plasma glucose level is associated with a $6 \%$ increased risk of hospitalization for pneumonia (32). Elevated blood glucose levels are also associated with altered activity of transporters responsible for clearing the lung of interstitial edema and for maintaining the integrity the pulmonary epithelial-endothelial barrier (33-35), both of which are likely to be important in determining the clinical outcome of SARS-CoV-2 infection. This hypothesis is 


\begin{tabular}{|c|c|c|c|c|c|c|}
\hline Site & Total & Cases & $\begin{array}{l}\% \\
\text { Cases / } \\
\text { Total }\end{array}$ & $\begin{array}{l}\text { Crude } \\
\text { Odds } \\
\text { Ratio }\end{array}$ & $\begin{array}{l}\text { Odds } \\
\text { Ratio }(95 \% \mathrm{Cl})\end{array}$ & $\begin{array}{l}\% \\
\text { Weight } \\
(I-V)\end{array}$ \\
\hline \multicolumn{7}{|l|}{ BMI $\geq 25-<30$} \\
\hline Norway & 189 & 12 & 6.3 & .51 & $0.72(0.33,1.59)$ & 4.52 \\
\hline Denmark, Copenhagen & 61 & 15 & 24.6 & 1.24 & $1.35(0.55,3.27)$ & 3.52 \\
\hline The Netherlands, Rotterdam ErasmusM & C62 & 16 & 25.8 & .62 & $0.58(0.24,1.42)$ & 3.54 \\
\hline The Netherlands, Rotterdam FG\&V & 62 & 10 & 16.13 & .72 & $0.74(0.22,2.46)$ & 1.92 \\
\hline The Netherlands, Amphia & 125 & 37 & 29.6 & .99 & $1.03(0.52,2.05)$ & 5.95 \\
\hline The Netherlands, CliniCo Consortium & 282 & 68 & 24.1 & .83 & $0.96(0.61,1.50)$ & 13.82 \\
\hline Austria & 42 & 3 & 7.1 & .23 & $0.29(0.06,1.39)$ & 1.13 \\
\hline Switzerland, Lausanne & 145 & 25 & 17.2 & 1.25 & $1.63(0.82,3.42)$ & 5.49 \\
\hline Switzerland, Ticino & 179 & 33 & 18.4 & .9 & $1.04(0.56,1.92)$ & 7.37 \\
\hline Italy, Milan Auxologico & 50 & 11 & 22 & .54 & $0.79(0.27,2.29)$ & 2.45 \\
\hline Italy, Milan Sacco & 111 & 14 & 12.6 & 1.29 & $1.15(0.45,2.95)$ & 3.16 \\
\hline Indonesia, Universitas Indonesia & 70 & 16 & 22.9 & 1.03 & $1.09(0.44,2.72)$ & 3.37 \\
\hline South Africa, Cape Town & 114 & 16 & 14 & .91 & $1.16(0.52,2.59)$ & 4.34 \\
\hline US, UCLA & 28 & 1 & 3.6 & .53 & $0.35(0.01,3.69)$ & 0.32 \\
\hline US, Saint Louis MO & 202 & 43 & 21.3 & .93 & $0.97(0.60,1.54)$ & 12.59 \\
\hline US, Cornell NY & 557 & 78 & 14 & .55 & $0.65(0.47,0.90)$ & 26.51 \\
\hline I-V Subtotal $\left(P^{2}=0.0 \%, P=0.651\right)$ & & & & & $0.88(0.74,1.04)$ & 100.00 \\
\hline$D+L$ Subtotal & & & & & $0.88(0.74,1.04)$ & \\
\hline \multicolumn{7}{|l|}{ BMI $\geq 30$} \\
\hline Norway & 117 & 7 & 6 & .48 & $1.00(0.37,2.69)$ & 3.12 \\
\hline Denmark, Copenhagen & 69 & 25 & 36.2 & 2.12 & $2.45(1.06,5.67)$ & 4.36 \\
\hline The Netherlands, Rotterdam ErasmusM & C56 & 9 & 16.1 & .34 & $0.35(0.13,0.94)$ & 3.13 \\
\hline The Netherlands, Rotterdam FG\&V & 66 & 7 & 10.61 & .44 & $0.46(0.12,1.75)$ & 1.71 \\
\hline The Netherlands, Amphia & 89 & 27 & 30.3 & 1.03 & $1.23(0.57,2.66)$ & 5.17 \\
\hline The Netherlands, CliniCo Consortium & 213 & 47 & 22.1 & .74 & $0.92(0.56,1.51)$ & 12.47 \\
\hline Austria & 26 & 5 & 19.2 & .71 & $1.33(0.24,7.25)$ & 1.06 \\
\hline Switzerland, Lausanne & 112 & 12 & 10.7 & .72 & $1.11(0.49,2.51)$ & 4.60 \\
\hline Switzerland, Ticino & 151 & 31 & 20.5 & 1.03 & $1.56(0.82,2.99)$ & 7.33 \\
\hline Italy, Milan Auxologico & 47 & 4 & 8.5 & .37 & $0.37(0.09,1.46)$ & 1.58 \\
\hline Italy, Milan Sacco & 56 & 11 & 19.6 & 2.18 & $2.47(0.89,6.87)$ & 2.94 \\
\hline Indonesia, Universitas Indonesia & 29 & 9 & 31 & 1.31 & $1.80(0.60,5.37)$ & 2.55 \\
\hline South Africa, Cape Town & 147 & 51 & 34.7 & 2.96 & $4.28(2.03,9.05)$ & 5.49 \\
\hline UCLA & 39 & 3 & 7.7 & 1.19 & $1.95(0.28,14.59)$ & 0.78 \\
\hline US, Saint Louis MO & 349 & 89 & 25.5 & 1.18 & $1.30(0.85,1.99)$ & 16.95 \\
\hline US, Cornell NY & 520 & 77 & 14.81 & .58 & $0.88(0.63,1.24)$ & 26.76 \\
\hline I-V Subtotal $\left(I^{2}=53.7 \%, P=0.006\right)$ & & & & & $1.17(0.98,1.39)$ & 100.00 \\
\hline \multirow[t]{2}{*}{ D+L Subtotal } & & & & & $1.23(0.92,1.64)$ & \\
\hline & & & &. & & \\
\hline
\end{tabular}

Figure 3-Meta-analysis odds ratios for in-hospital mortality by BMI category. Models were adjusted for age ( $<65, \geq 65$ years), sex (male/female), preexisting cardiovascular disease (yes/no), diabetes (yes/no), preexisting respiratory conditions (yes/no), and hypertension (yes/no). The reference BMI is in the normal range (i.e., $\geq 18$ to $<25$ ). The $95 \% \mathrm{Cls}$ of the odds ratios were not adjusted for multiple testing and should not be used to infer definitive effects. Data from Guandong Province, China, and Singapore were not available for in-hospital mortality. D+L, DerSimonian and Laird random effects model; FG\&V, Franciscus Gasthuis \& Vlietland; I-V, inverse-variance weighted fixed effects model; US UCLA, University of California, Los Angeles, California.

consistent with observations that wellcontrolled blood glucose levels correlated with improved clinical outcomes in patients with COVID-19 who also had diabetes (36). More severe COVID-19, however, may also be associated with elevated glucose levels (37). With respect to the relationship between longer-term glucose control and COVID-19 severity, an elevated hemoglobin $A_{1 c}$ is associated with increased risk of hospital admission due to COVID-19 in those with diabetes (38). Additional studies investigating the mechanistic roles of both $\mathrm{BMI}$ and diabetes in COVID-19 severity are warranted.
Currently, it is estimated that $90 \%$ of patients with type 2 diabetes are overweight or obese (39). Previous studies have suggested that among patients with COVID-19 who have diabetes, nonsurvivors had a greater prevalence of comorbidities compared with survivors (25). Given the clear independent role of BMI in COVID-19 severity, we reasoned that patients with both diabetes and an elevated BMI may be at increased risk of severe disease outcomes compared with patients with diabetes and a BMI in the normal range. Surprisingly, BMI was not associated with the risk of in-hospital respiratory support or death among patients with both COVID-19 and diabetes. Larger studies will be needed to confirm these findings; however, this finding may reflect a "threshold effect" of susceptibility to severe COVID-19 in these conditions. This hypothesis will require clinical and experimental evaluation.

Our data will inform public policy, particularly for risk-stratification of severe COVID-19 disease. The U.S. Centers for Disease Control and Prevention identifies individuals with obesity ( $\mathrm{BMI} \geq 30$ ) as being at increased risk for severe disease, as well as those with cardiovascular 


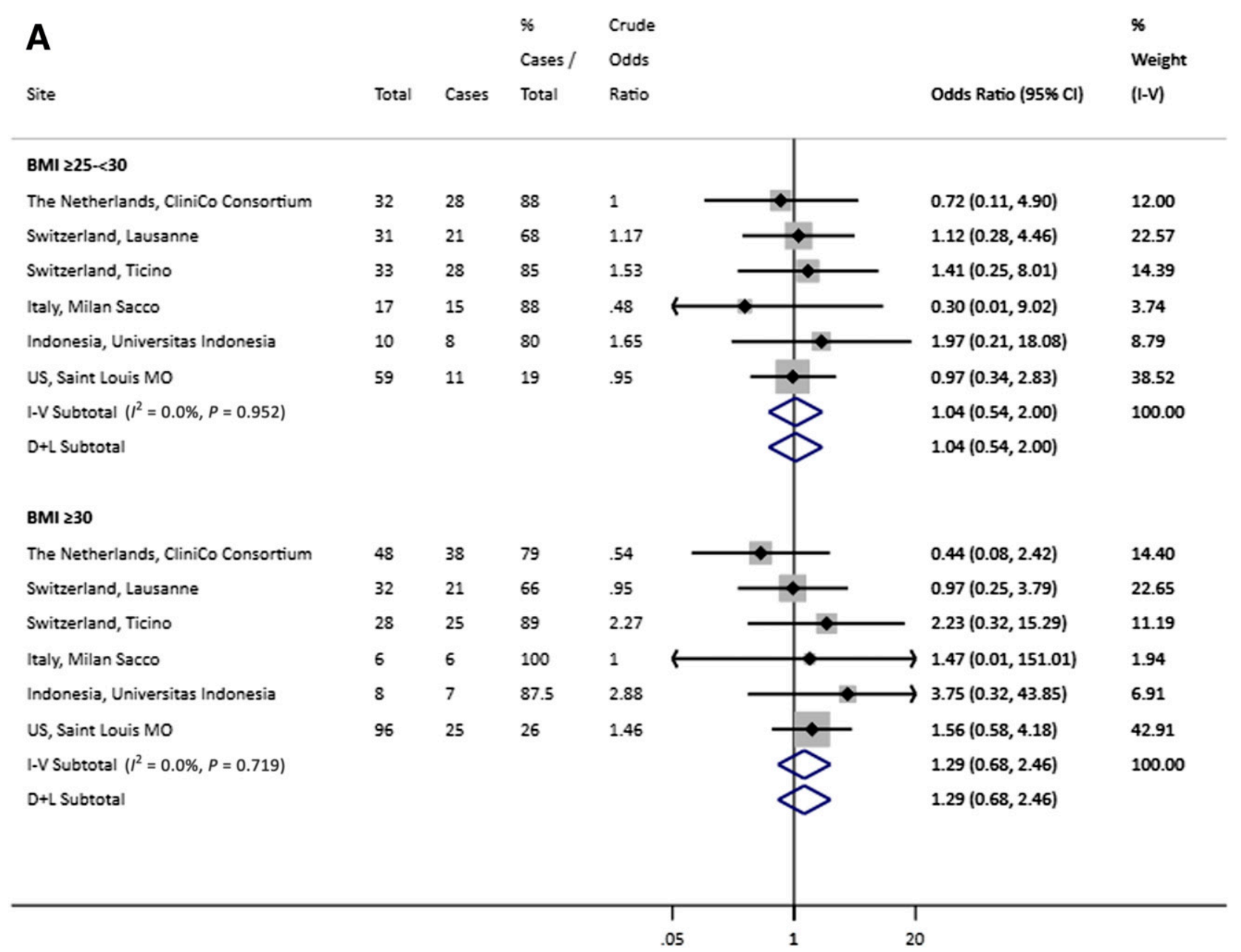

Figure 4-Meta-analysis odds ratios for supplemental oxygen/noninvasive ventilatory support $(A)$, invasive mechanical ventilatory support $(B)$, and in-hospital mortality by BMI category in patients with preexisting diabetes $(C)$. Models were adjusted for age ( $<65, \geq 65$ years), sex (male/female), preexisting cardiovascular disease (yes/no), preexisting respiratory conditions (yes/no), and hypertension (yes/no). The reference BMI is in the normal range (i.e., $\geq 18$ to $<25$ ). The $95 \% \mathrm{Cl}$ of the odds ratios have not been adjusted for multiple testing and should not be used to infer definitive effects. Data from New York were not available for this subgroup analysis. D + L, DerSimonian and Laird random effects model; FG\&V, Franciscus Gasthuis \& Vlietland; $\mathrm{I}-\mathrm{V}$, inverse-variance weighted fixed effects model.

disease, and has recently outlined that individuals who are overweight may be at increased risk (40). Similarly, the most recent guidelines from Public Health England consider overweight and obesity as risk factors for severe COVID-19(41), in contrast to more conservative guidelines from the UK National Health Service that suggested an increased risk only for a BMI of $\geq 40$ (42). The World Health Organization now considers obesity a risk factor for severe COVID-19 disease (43). Inconsistent recommendations may impede optimal patient care and compromise clear public health messaging. To our knowledge, there is currently no clinical guidance on the role of BMI in COVID19 risk stratification of patients with diabetes.
We acknowledge limitations of our study. Data on socioeconomic status were not available, limiting the interpretation of these findings, particularly because there may be important relationships among $\mathrm{BMI}$, race/ethnicity, and socioeconomic status (44). Adjustment for confounders including smoking and race/ethnicity was only possible for five sites, with no difference in odds ratio observed. Supplemental oxygen use varied; oxygen was administered to all hospitalized patients at a limited number of sites, affecting our ability to determine the influence of host comorbidities on this outcome. There may also be varying and unmeasurable differences in thresholds for escalating care in those with overweight and obesity.
Given this analysis involves patients admitted to hospital with COVID-19 only, we also were unable to assess whether patients with diabetes and obesity were more likely to experience out-of-hospital death due to COVID-19 infection. These patients were not captured in the data, and this may have resulted in an underestimation of overall mortality. At some sites, BMI was not consistently recorded during the study period, which may have introduced site-specific bias. Because of the relatively small numbers of patients at some sites, we were unable to stratify $\mathrm{BMI}$ categories to include underweight (BMI $<18.5$ ) or $\mathrm{BMI}>40$, so we were unable to report specific odds ratios for these groups. We were unable to 


\begin{tabular}{|c|c|c|c|c|c|c|}
\hline $\mathbf{B}$ & Total & Cases & $\begin{array}{l}\% \\
\text { Cases / } \\
\text { Total }\end{array}$ & $\begin{array}{l}\text { Crude } \\
\text { Odds } \\
\text { Ratio }\end{array}$ & Odds Ratio (95\% Cl) & $\begin{array}{l}\% \\
\text { Weight } \\
(1-V)\end{array}$ \\
\hline \multicolumn{7}{|l|}{ BMI $225-<30$} \\
\hline Norway & 20 & 3 & 15 & 1.25 & $1.18(0.22,6.41)$ & 9.08 \\
\hline The Netherlands, Rotterdam FG\&V & 12 & 4 & 33 & 1.5 & $3.84(0.21,70.68)$ & 3.05 \\
\hline The Netherlands, Clinico Consortium & 40 & 8 & 20 & .8 & $0.63(0.16,2.46)$ & 13.83 \\
\hline Switzerland, Lausanne & 32 & 2 & 6 & 1.2 & $0.98(0.07,13.35)$ & 3.75 \\
\hline Switzerland, Ticino & 37 & 4 & 11 & .85 & $0.77(0.11,5.42)$ & 6.80 \\
\hline Italy, Milan Sacco & 15 & 4 & 27 & 4.3 & $3.08(0.10,92.47)$ & 2.23 \\
\hline Indonesia, Universitas Indonesia & 11 & 3 & 27 & .56 & $0.48(0.07,3.25)$ & 7.01 \\
\hline South Africa, Cape Town & 29 & 1 & 3 & .57 & $0.84(0.07,10.59)$ & 4.10 \\
\hline US, Saint Louis MO & 80 & 21 & 26 & .51 & $0.51(0.25,1.05)$ & 50.15 \\
\hline I-v Subtotal $\left(l^{2}=0.0 \%, P=0.907\right)$ & & & & & $0.67(0.40,1.12)$ & 100.00 \\
\hline D+L Subtotal & & & & & $0.67(0.40,1.12)$ & \\
\hline \multicolumn{7}{|l|}{ BMI 230} \\
\hline Norway & 21 & 4 & 19 & 1.57 & $1.31(0.22,7.49)$ & 7.56 \\
\hline The Netherlands, Rotterdam FG\&V & 18 & 6 & 33 & 1.5 & $1.34(0.09,20.16)$ & 3.21 \\
\hline The Netherlands, Clinico Consortium & 56 & 8 & 14 & .53 & $0.59(0.15,2.37)$ & 12.35 \\
\hline Switzerland, Lausanne & 42 & 10 & 24 & 5.63 & $6.35(0.65,61.81)$ & 4.54 \\
\hline Switzerland, Ticino & 35 & 7 & 20 & 1.75 & $1.42(0.23,8.76)$ & 7.10 \\
\hline Italy, Milan Sacco & 6 & 2 & 33 & 6.11 & $5.17(0.16,167.44)$ & 1.95 \\
\hline Indonesia, Universitas Indonesia & 7 & 3 & 43 & .6 & $0.59(0.06,5.41)$ & 4.64 \\
\hline South Africa, Cape Town & 57 & 14 & 25 & 5.21 & $7.83(1.27,48.32)$ & 7.11 \\
\hline US, Saint Louis MO & 136 & 40 & 29 & .6 & $0.57(0.29,1.12)$ & 51.54 \\
\hline I-v Subtotal $\left(I^{2}=33.1 \%, P=0.153\right)$ & & & & & $0.94(0.58,1.53)$ & 100.00 \\
\hline D+L Subtotal & & & & & $1.25(0.62,2.53)$ & \\
\hline
\end{tabular}

Figure 4-Continued.

differentiate between type 1 and type 2 diabetes from the data available. Notwithstanding, the majority of patients with diabetes included would be expected to have type 2 diabetes, given the expected prepandemic relative prevalence (25). Moreover, type 1 diabetes has not been associated with increased severity of COVID-19 (45); therefore, we believe the findings for patients with diabetes predominantly represent those with type 2 diabetes. It is important to note that the modest sample size of this study precludes precise estimates of risk, particularly with respect to the associations among the subgroup of patients with diabetes. We acknowledge that the number of deaths likely decreased over the period of the study, which may have altered results dependent on dates of data collection and the timing of COVID-
19 surges in different countries. Given that improvements in clinical care did not occur uniformly in all countries, however, we were unable to adjust for this in our analysis. Finally, although we enrolled multiple sites, our findings should not be considered regionally or globally representative and the study population was underrepresented for low- and middle-income countries, which may limit generalizability.

Notwithstanding, to our knowledge, this study remains one of the largest multinational study to date on the risk factors associated with severe COVID-19. Inclusion of individuals from low- and middle-income countries and disadvantaged or higher-risk populations in such analyses is essential, and it is hoped that as the pandemic progresses and more data become available, data from these populations can be added to our ongoing analysis; potential collaborators are encouraged to contact the corresponding authors.

In conclusion, our findings highlight the importance of maintaining a healthy BMI, because patients with either overweight or obesity are at risk for severe COVID-19. Although reducing the current levels of overweight and obesity is unlikely in the short term to have an impact on the COVID-19 pandemic, doing so may contribute to reducing disease burden in future viral pandemics $(41,46)$. Furthermore, the absence of an association between overweight/obesity and COVID-19 severity among those with diabetes should guide additional exploration of mechanistic pathways and may inform risk stratification and appropriate patient treatment. Finally, our findings 


\begin{tabular}{|c|c|c|c|c|c|c|}
\hline $\mathrm{C}$ & Total & Cases & $\begin{array}{l}96 \\
\text { Cases / } \\
\text { Total }\end{array}$ & $\begin{array}{l}\text { Crude } \\
\text { Odds } \\
\text { Ratio }\end{array}$ & $\begin{array}{l}\text { Odds } \\
\text { Ratio (95\% Cl) }\end{array}$ & $\begin{array}{l}\% \\
\text { Weight } \\
(1-V)\end{array}$ \\
\hline \multicolumn{7}{|l|}{$B M 1225-<30$} \\
\hline Norway & 22 & 2 & 9 & .9 & $0.81(0.08,7.94)$ & 3.35 \\
\hline The Netherlands, Rotterdam ErasmusMC & 15 & 4 & 27 & .36 & $0.66(0.07,6.28)$ & 3.50 \\
\hline The Netherlands, Rotterdam FG\&V & 16 & 4 & 25 & .89 & $0.32(0.01,7.61)$ & 1.61 \\
\hline The Netherlands, Amphia & 25 & 8 & 32 & 1.18 & $0.94(0.11,7.50)$ & 3.97 \\
\hline The Netherlands, Clinico Consortium & 57 & 17 & 30 & .64 & $0.65(0.25,1.70)$ & 19.26 \\
\hline Switzerland, Lausanne & 41 & 9 & 22 & 1.34 & $1.83(0.44,7.83)$ & 8.53 \\
\hline Switzerland, Ticino & 47 & 10 & 21 & .62 & $0.69(0.21,2.29)$ & 12.40 \\
\hline Italy, Milan Sacco & 17 & 3 & 18 & .89 & $0.64(0.05,8.76)$ & 2.59 \\
\hline Indonesia, Universitas Indonesia & 13 & 5 & 38 & .44 & $0.44(0.08,2.29)$ & 6.50 \\
\hline South Africa, Cape Town & 35 & 6 & 17 & 1.66 & $2.10(0.51,8.69)$ & 8.80 \\
\hline US, Saint Louis MO & 95 & 15 & 16 & .67 & $0.67(0.31,1.46)$ & 29.48 \\
\hline I-V Subtotal $\left(I^{2}=0.0 \%, P=0.930\right)$ & & & & & $0.79(0.52,1.20)$ & 100.00 \\
\hline$D+$ L Subtotal & & & & & $0.79(0.52,1.20)$ & \\
\hline \multicolumn{7}{|l|}{ BMI 230} \\
\hline Norway & 24 & 3 & 12 & 1.29 & $1.71(0.20,14.49)$ & 3.56 \\
\hline The Netherlands, Rotterdam ErasmusMC & 11 & 2 & 18 & .22 & $0.28(0.03,2.44)$ & 3.38 \\
\hline The Netherlands, Rotterdam FG\&V & 20 & 2 & 10 & .3 & $0.06(0.00,2.45)$ & 1.07 \\
\hline The Netherlands, Amphia & 25 & 9 & 36 & 1.41 & $2.13(0.23,20.13)$ & 3.27 \\
\hline The Netherlands, Clinico Consortium & 73 & 17 & 23 & .46 & $0.51(0.20,1.35)$ & 17.91 \\
\hline Switzerland, Lausanne & 46 & 4 & 9 & .45 & $1.09(0.21,5.72)$ & 6.00 \\
\hline Switzerland, Ticino & 48 & 13 & 27 & .85 & $1.16(0.36,3.77)$ & 11.84 \\
\hline Italy, Milan Sacco & 6 & 1 & 17 & 1 & $1.67(0.09,31.86)$ & 1.90 \\
\hline Indonesia, Universitas Indonesia & 8 & 3 & 37.5 & .42 & $0.47(0.08,2.94)$ & 4.92 \\
\hline South Africa, Cape Town & 76 & 31 & 41 & 5.51 & $9.41(2.61,33.96)$ & 9.92 \\
\hline US, Saint Louis MO & 184 & 48 & 26 & 1.27 & $1.25(0.64,2.45)$ & 36.24 \\
\hline I-V Subtotal $\left(I^{2}=45.5 \%, P=0.050\right)$ & & & & & $1.16(0.77,1.74)$ & 100.00 \\
\hline$D+L$ Subtotal & & & & & $1.14(0.61,2.13)$ & \\
\hline
\end{tabular}

Figure 4-Continued.

may inform immunization prioritization for higher-risk groups.

Acknowledgments. Authors from several sites wish to extend acknowledgments: Those at Auxilogico, Milan, Italy, acknowledge Drs Irene Campi, lacopo Chiodini, Luca Giovanelli, Giovanni Perego, Francesca Heilbron, Roberto Menè, Andrea Cascella, Stefano Vicini, and all nurses; those at the University of California, Los Angeles, acknowledge Dr. Paul C. Adamson; those in Cape Town, South Africa, acknowledge doctors and nurses working in COVID-19 inpatient service; those in Indonesia thank the patients, doctors, nurses, pharmacists, other health care workers, and research administrators at all the participating sites. Authors in Ticino, Switzerland, acknowledge Lorenzo Ruinelli; those in Lausanne, Switzerland, acknowledge Orio Manuel, Desgranges Florian, Filippidis Paraskevas, Kampouri Eleftheria-Evdokia, Tschopp Jonathan, and Viala Benjamin; those at Amphia, the Netherlands, acknowledge A.G. Loman, B.W. Driessen, and Franciscus Gasthuis; those in Vlietland, Rotterdam, the Netherlands, acknowledge Dr. Bianca M. Boxma-de Klerk; those at Washington University,
St. Louis, Missouri, acknowledge Drs. Albert Lai and Randi Foraker of the Institute for Informatics at Washington University School of Medicine. The main writing group acknowledges patients and their families and health care providers worldwide.

Funding. There was no specific project funding for the study. Individual investigators were funded as follows: J.E.M. was supported by a fellowship from the DHB Foundation, Australia; S.B. is supported by the Dutch Heart Foundation (Dekker grant 2018-T028); E.M. is supported by the World Health Organization Collaborating Centre for Reference and Research on Influenza, funded by the Australian Commonwealth Government, Department of Health. K.R.S. was supported by the Australian Research Council (grant DE180100512); A.N. is supported by a Health Research Board of Ireland Clinical Trail Network award (grant CTN-2014-012); and D.P.B. was supported by a National Health and Medical Research Council Australian Investigator grant (GTN1175744). Research at the Murdoch Children's Research Institute is supported by the Victorian Government's Operational Infrastructure Support Program.
The funders had no role in study design, data collection, data analysis, data interpretation, or writing of the report.

Duality of Interest. No potential conflicts of interest relevant to this article were reported. Author Contributions. Authors had full access to their corresponding site's data in the study and had final responsibility for the decision to submit for publication. D.K.L., E.M., Y.Z., K.R.S., and D.P.B. contributed to data interpretation and wrote the first draft of the manuscript. J.E.M., S.B., and C.S. performed the data analysis, contributed to data interpretation, and reviewed the manuscript. R.S., A.N., and G.C. contributed to the data interpretation and reviewed the manuscript. J.E.M. and D.P.B. are the guarantors of this work and, as such, had full access to all the data in the study and take responsibility for the integrity of the data and the accuracy of the data analysis.

\section{References}

1. World Health Organization. Coronovirus Disease (COVID-19) Situation Report -162. Geneva, World Health Organization, 2020, p. 17

2. Popkin BM, Du S, Green WD, et al. Individuals with obesity and COVID-19: a global perspective 
on the epidemiology and biological relationships. Obes Rev 2020;21:e13128

3. Selvin E, Juraschek SP. Diabetes epidemiology in the COVID-19 pandemic. Diabetes Care 2020; 43:1690-1694

4. Rottoli M, Bernante P, Belvedere A, et al. How important is obesity as a risk factor for respiratory failure, intensive care admission and death in hospitalised COVID-19 patients? Results from a single Italian centre. Eur J Endocrinol 2020;183: 389-397

5. Simonnet $A$, Chetboun M, Poissy J, et al.; LICORN and the Lille COVID-19 and Obesity study group. High prevalence of obesity in severe acute respiratory syndrome coronavirus-2 (SARS-CoV2) requiring invasive mechanical ventilation. Obesity (Silver Spring) 2020;28:1195-1199

6. Halasz G, Leoni ML, Villani GQ, Nolli M, Villani $M$. Obesity, overweight and survival in critically ill patients with SARS-CoV-2 pneumonia: is there an obesity paradox? Preliminary results from Italy. Eur J Prev Cardiol. 7 July 2020 [Epub ahead of print]. DOI: 10.1177/2047487320939675

7. Hamer M, Gale CR, Kivimäki M, Batty GD. Overweight, obesity, and risk of hospitalization for COVID-19: a community-based cohort study of adults in the United Kingdom. Proc Natl Acad Sci U S A 2020;117:21011-21013

8. Tartof SY, Qian L, Hong V, et al. Obesity and mortality among patients diagnosed with COVID19: results from an integrated health care organization. Ann Intern Med 2020;173:773-781

9. Nakeshbandi M, Maini R, Daniel P, et al. The impact of obesity on COVID-19 complications: a retrospective cohort study. Int J Obes 2020;44: 1832-1837

10. Petrilli CM, Jones SA, Yang J, et al. Factors associated with hospital admission and critical illness among 5279 people with coronavirus disease 2019 in New York City: prospective cohort study. BMJ 2020;369:m1966

11. World Health Organization. Obesity and overweight, 2020. Accessed 17 October 2020. Available from https://www.who.int/news-room/ fact-sheets/detail/obesity-and-overweight

12. World Health Organization. Prevalence of obesity among adults, BMI $>=30$ (crude estimate) (\%). 2017. Accessed 19 October 2020. Available from https://www.who.int/data/gho/ data/indicators/indicator-details/GHO/prevalenceof-obesity-among-adults-bmi- $=-30$-(crudeestimate)-(-)

13. Smith KB, Smith MS. Obesity statistics. Prim Care 2016;43:121-135, ix

14. World Health Organization Expert Consultation. Appropriate body-mass index for Asian populations and its implications for policy and intervention strategies. Lancet 2004;363:157163

15. Harris R, Bradburn M, Deeks J, Harbord R, Altman $D$, Sterne J. metan: Fixed- and randomeffects meta-analysis. Stata J 2008;8:3-28 16. RStudio. Integrated Development for R. RStudio, 2020. Accessed 3 September 2020. Available from https://www.rstudio.com/
17. Statacorp. Stata Statistical Software: release 16. College Station, TX, StataCorp LLC, 2019 18. Zaki N, Alashwal H, Ibrahim S. Association of hypertension, diabetes, stroke, cancer, kidney disease, and high-cholesterol with COVID-19 disease severity and fatality: a systematic review. Diabetes Metab Syndr 2020;14:1133-1142 19. Williamson EJ, Walker AJ, Bhaskaran K, et al. Factors associated with COVID-19-related death using OpenSAFELY. Nature 2020;584:430436

20. Anderson MR, Geleris J, Anderson DR, et al. Body mass index and risk for intubation or death in SARS-CoV-2 infection: a retrospective cohort study. Ann Intern Med 2020;173:782-790

21. Klang E, Kassim G, Soffer S, Freeman R, Levin $M A$, Reich DL. Severe obesity as an independent risk factor for COVID-19 mortality in hospitalized patients younger than 50 . Obesity (Silver Spring) 2020;28:1595-1599

22. Watanabe M, Caruso D, Tuccinardi D, et al. Visceral fat shows the strongest association with the need of intensive care in patients with COVID19. Metabolism 2020;111:154319

23. Monteiro AC, Suri R, Emeruwa 10 , et al. Obesity and smoking as risk factors for invasive mechanical ventilation in COVID-19: a retrospective, observational cohort study. PLoS One 2020; 15: 0238552

24. Cariou B, Hadjadj S, Wargny M, et al.; CORONADO Investigators. Phenotypic characteristics and prognosis of inpatients with COVID-19 and diabetes: the CORONADO study. Diabetologia 2020;63:1500-1515

25. Shi $Q$, Zhang $X$, Jiang $F$, et al. Clinical characteristics and risk factors for mortality of COVID-19 patients with diabetes in Wuhan, China: a two-center, retrospective study. $\mathrm{Di}$ abetes Care 2020;43:1382-1391

26. Suganami T, Tanaka M, Ogawa Y. Molecular mechanisms underlying obesity-induced chronic inflammation. In Chronic Inflammation: Mechanisms and Regulation. Miyasaka M, Takatsu K, Eds. Tokyo, Springer Japan, 2016, pp. 291-298 27. Siegers JY, Novakovic B, Hulme KD, et al. A high-fat diet increases influenza $A$ virus-associated cardiovascular damage. J Infect Dis 2020; 222:820-831

28. Smith AG, Sheridan PA, Tseng RJ, Sheridan JF, Beck MA. Selective impairment in dendritic cell function and altered antigen-specific CD8 + T-cell responses in diet-induced obese mice infected with influenza virus. Immunology 2009;126:268279

29. Lockhart SM, O'Rahilly S. When two pandemics meet: why is obesity associated with increased COVID-19 mortality? Med (NY) 2020;1: 33-42

30. Paich HA, Sheridan PA, Handy J, et al. Overweight and obese adult humans have a defective cellular immune response to pandemic H1N1 influenza A virus. Obesity (Silver Spring) 2013;21: 2377-2386

31. Kassir R. Risk of COVID-19 for patients with obesity. Obes Rev 2020;21:e13034
32. Benfield $T$, Jensen JS, Nordestgaard BG. Influence of diabetes and hyperglycaemia on infectious disease hospitalisation and outcome. Diabetologia 2007;50:549-554

33. Yamashita $T$, Umeda $F$, Hashimoto $T$, et al. Effect of glucose on Na, K-ATPase activity in cultured bovine aortic endothelial cells. Endocrinol Jpn 1992;39:1-7

34. Rivelli JF, Amaiden MR, Monesterolo NE, et al. High glucose levels induce inhibition of $\mathrm{Na}$,K-ATPase via stimulation of aldose reductase, formation of microtubules and formation of an acetylated tubulin/Na,K-ATPase complex. Int $J$ Biochem Cell Biol 2012;44:1203-1213

35. Hulme KD, Yan L, Marshall RJ, et al. High glucose levels increase influenza-associated damage to the pulmonary epithelial-endothelial barrier. elife 2020;9:e56907

36. Zhu L, She Z-G, Cheng X, et al. Association of blood glucose control and outcomes in patients with COVID-19 and pre-existing type 2 diabetes. Cell Metab 2020;31:1068-1077.e3

37. Coppelli A, Giannarelli R, Aragona M, et al.; Pisa COVID-19 Study Group. Hyperglycemia at hospital admission is associated with severity of the prognosis in patients hospitalized for COVID19: the Pisa COVID-19 Study. Diabetes Care 2020; 43:2345-2348

38. Merzon E, Green I, Shpigelman M, et al. Haemoglobin A1c is a predictor of COVID-19 severity in patients with diabetes. Diabetes Metab Res Rev. 27 August 2020 [Epub ahead of print]. DOI: $10.1002 / d m r r .3398$

39. Public Health England. Adult Obesity and Type 2 Diabetes. London, Public Health England, 2014, pp. 39

40. Centers for Disease Control and Prevention. People with certain medical conditions, 2020. Accessed 26 October 2020. Available from https:// www.cdc.gov/coronavirus/2019-ncov/needextra-precautions/people-with-medical-conditions .html

41. Blackshaw J, Feeley A, Mabbs L, et al. Excess Weight and COVID-19: Insights from New Evidence. London, Public Health England, 2020

42. National Health Service. Coronavirus (COVID19): Shielded patients list, 2020. Accessed 13 July 2020. Available from https://digital.nhs.uk/ coronavirus/shielded-patient-list\#risk-criteria 43. World Health Organization. COVID-19: vulnerable and high risk groups, 2020. Accessed 12 July 2020 . Available from https://www.who .int/westernpacific/emergencies/covid-19/ information/high-risk-groups

44. McLaren L. Socioeconomic status and obesity. Epidemiol Rev 2007;29:29-48

45. Vangoitsenhoven R, Martens PJ, van Nes F, et al. No evidence of increased hospitalization rate for COVID-19 in community-dwelling patients with type 1 diabetes. Diabetes Care 2020; 43:e118-e119

46. Short KR, Kedzierska K, van de Sandt CE. Back to the future: lessons learned from the 1918 influenza pandemic. Front Cell Infect Microbiol 2018;8:343 